\title{
Use of Non-Occupational Post-Exposure Prophylaxis does not Lead to an Increase in High Risk Sex Behaviors in Men Who have Sex with Men Participating in the EXPLORE Trial
}

\author{
Deborah Donnell • Matthew J. Mimiaga • \\ Kenneth Mayer • Margaret Chesney • \\ Beryl Koblin • Thomas Coates \\ Published online: 19 May 2010 \\ (c) The Author(s) 2010. This article is published with open access at Springerlink.com
}

\begin{abstract}
Non-occupational post-exposure prophylaxis (nPEP) use is an HIV prevention strategy that has been recommended by the CDC to prevent HIV infection after a high risk sexual exposure since 1997. In a behavioral intervention trial of 4,295 MSM we assessed perceptions and use of nPEP over 4 years in six cities across the United States. Overall, $1.9 \%$ of MSM reported use of nPEP prior to enrollment, and $6.3 \%$ at least once during the trial. Awareness of nPEP was reported by $47.5 \%$, with higher awareness in two sites with funded nPEP programs. Three seroconversions occurred in the 384 visits where nPEP
\end{abstract}

D. Donnell $(\square)$

Vaccine and Infectious Disease Institute, Fred Hutchinson Cancer Research Ctr, 1100 Fairview Ave N,

PO Box 10924, Seattle, WA 98109, USA

e-mail: deborah@scharp.org

M. J. Mimiaga

Harvard Medical School/Massachusetts General Hospital,

Boston, MA, USA

\section{J. Mimiaga · K. Mayer}

The Fenway Institute, Fenway Community Health,

Boston, MA, USA

M. Chesney

Osher Center for Integrative Medicine, University of California,

San Francisco, CA, USA

B. Koblin

The New York Blood Center, New York, NY, USA

T. Coates

Department of Medicine, Division of Infectious Diseases, David Geffen School of Medicine, University of California, Los Angeles, CA, USA courses were reported, with no effect of nPEP on risk of HIV acquisition in this cohort (hazard ratio $=0.91,95 \%$ confidence interval $[0.29,2.86])$. NPEP users were a riskier group: increased odds of nPEP use were observed in association with multiple partners and unprotected receptive and insertive anal sex with HIV infected partners and partners with unknown HIV status. NPEP use was also associated with use of illicit drugs (injection drugs, crack cocaine, hallucinogens, and amphetamines). Importantly, willingness to use nPEP after high risk sex was associated with lower odds of high risk sex. After an episode of nPEP use, nPEP users remained more likely to report high risk sex than those in this cohort who had not previously used nPEP. However, within the subset of people who had previously reported high risk sex, previous nPEP use was not associated with higher odds of high risk sex, thus allaying fears that availability of nPEP would lead to an increase in high risk sex.

Keywords MSM $\cdot$ HIV $~$ PEP $\cdot$ Behavioral disinhibition

\section{Introduction}

Non-occupational post-exposure prophylaxis (nPEP) is an emergency medical response where antiretroviral drugs are given to prevent HIV infection after high risk sexual or injection drug use exposure [1-3]. For greatest potential efficacy, nPEP should be initiated within 2-24 h (and no later than $48-72 \mathrm{~h}$ ) of possible exposure to HIV and must continue for 28 days [2]. Although nPEP has not been conclusively proven to prevent the acquisition of HIV infection, human and animal clinical and observational studies suggest that if medication is initiated quickly 
after possible exposure, it may prevent HIV acquisition [2-9].

Studies of occupational post-exposure prophylaxis (PEP) provide strong support for efficacy in reducing HIV transmission risk. For example, a multi-site international case-control study of health care workers exposed to HIVinfected blood found an $80 \%$ reduction in the odds of infection for those individuals who received PEP [10]. Studies examining use of non-occupational PEP after sexual assault suggest the treatment might reduce risk of infection following sexual HIV exposure [11, 12], and a trial of take home nPEP in Brazilian men who have sex with men (MSM) reported nPEP users were much less likely to seroconvert after engaging in risky sexual practices than those who did not use nPEP [5].

NPEP is not widely utilized after high risk sexual exposure in the United States. Between 1998 and 2004 the national US nPEP register received approximately 800 nPEP reports; although it is believed that the majority of clinicians did not report to the registry [3]. Fenway Community Health, which serves a large proportion of MSM in the Boston area, prescribes an average of 60 courses of nPEP each year [13]. San Francisco clinics with nPEP studies reported about 400 nPEP patients in 2.5 years [3]. One multi-site HIV vaccine study found that only two percent of 5,418 participants (majority MSM) had reported nPEP use during the study, although $46 \%$ had heard of nPEP [14]. Predictors of nPEP use among trial participants included higher educational level, greater recreational drug use, study enrollment in California, and having a known HIV-infected partner. A cross sectional study of gay and bisexual men in San Francisco found slightly higher levels of use (4\%) but similar levels of awareness [15].

When nPEP became widely available in the mid-to-late 1990s, clinical and public health communities raised concerns that a perception that nPEP could prevent HIV infection might increase the level or frequency of high risk sexual and injection drug behaviors [16]. Some studies have suggested that nPEP users may be recurrent sexual risk takers and since HIV seroconversions among nPEP users have occurred $[17,18]$, it is known nPEP may only be partially effective. However, to date no studies have found that use or awareness of nPEP has led to increase in highrisk sexual behavior among MSM [5, 17-22].

In this study of nPEP use in a large multi-site longitudinal study of high risk MSM, we examined knowledge and use of nPEP and its association with HIV risk behaviors, including risk of HIV seroconversion. We assessed the impact of nPEP on risk disinhibition before and after nPEP use and characterized the sexual risk of nPEP users using assessments of nPEP use over a 4 year period.

\section{Methods}

Participants and Procedures

The EXPLORE study was a randomized HIV-prevention trial among 4295 MSM conducted in six U.S. cities between January 1999 and February 2003 [23]. Of the six cities, San Francisco and Boston had funded nPEP initiatives associated with the study site, whereas Seattle, Chicago, New York and Denver did not. Men, 16 years or older, were eligible to enroll if they were HIV-uninfected and reported anal sex with another man during the past year, excluding those in a mutually monogamous relationship for the past 2 years with an HIV-uninfected male partner [24]. Men were randomized to receive a behavioral intervention versus standard risk reduction counseling. The experimental intervention consisted of 10 core counseling modules delivered over 6 months in one-on-one counseling sessions with subsequent maintenance sessions every 3 months [25]. The intervention was designed to address individual, interpersonal, and situation-related factors associated with risk taking among men who have sex with men, such as greater pleasure in or enjoyment of riskrelated sexual behavior, negative mood states, communication difficulties, social norms encouraging misperceptions of risk and risk taking, use of alcohol or recreational drugs, and life events and environments that are catalysts for risk taking. The standard risk reduction counseling was modeled after Project RESPECT [26]. Participants in both arms were tested for HIV every 6 months and completed a sexual risk behavior and psychosocial assessment questionnaire using audio-computer-assisted self-interview (ACASI). Additional methods for data collection of the EXPLORE cohort have been described previously [23-25].

\section{Measures}

The primary outcome in EXPLORE was HIV seroconversion: blood samples were collected for HIV at each 6 month follow-up visit over the study's duration. Antibodies to HIV were detected by ELISA. Serum samples shown to be reactive after a first test were retested in duplicate. Repeatedly reactive samples were confirmed by western-blot assay or immunofluorescence.

Knowledge about nPEP, willingness to use nPEP, access to nPEP, nPEP use and perceptions about disinhibition were assessed at baseline and each scheduled visit using ACASI. Knowledge about nPEP was assessed at baseline: "Have you read or heard about the idea of HIV-negative people taking anti-HIV medications right before or after a high-risk exposure, to keep from getting infected with HIV?". Willingness to use nPEP was assessed at each visit by whether participants were "very likely," "somewhat 
likely," or "not at all likely," to try anti-HIV medications to prevent HIV infection after unprotected receptive anal sex with an HIV-positive partner. NPEP access was assessed through Yes/No responses to "If you had a highrisk exposure and wanted to get anti-HIV medications to try to prevent HIV infection and cost was not an issue, do you think you would be able to easily obtain the drugs?". Lifetime NPEP use was assessed at baseline by responses to "Have you ever used anti-HIV medications to prevent HIV infection either before or after a high-risk sexual or drug use exposure?"; at follow-up visits, participants reported any nPEP use since the last visit. Lastly, perception about nPEP and disinhibition were recorded on a 6 point Likert scale from strongly, somewhat, or slightly disagree, to slightly, somewhat, or strongly agree with the statement "Easy access to PEP will increase unsafe sex among people I know."

Non-prescription drug use in the last 6 months was coded as any use of each of marijuana, poppers, cocaine, amphetamines, hallucinogens, and injection drugs. Current alcohol use was categorized as "none," "light," (three or less drinks/day on no more than 1-2 days/week) "moderate," (four or five drinks/day on no more than 1-2 days/ week, or one to five drinks/day on 3-6 days/week, or one to three drinks/day on a daily basis) or "heavy" (four or more drinks every day or six or more drinks on a typical day when drinking).

High risk HIV sexual behaviors were any unprotected anal sex in the previous 6 months, categorized by reported HIV status of partners: HIV-infected (HIV+), unknown HIV status(HIV-unk) or HIV uninfected (HIV-); and unprotected receptive(URA) or insertive(UIA) anal sex, resulting in six sexual risk variables: URA with $\mathrm{HIV}+$, i.e., any unprotected receptive anal sex with an HIV-infected partner; UIA with HIV+; URA with HIV-unk; UIA with HIV-unk; URA with HIV-; UIA with HIV-. We also created a composite sexual risk measure, serodiscordant unprotected anal sex (SDUA), defined as any unprotected receptive or insertive anal sex with HIV-infected or unknown HIV status partners.

\section{Statistical Methods}

Association of baseline nPEP questions with demographic characteristics was assessed using Chi-squared tests for site and race/ethnicity, and test for trend for age and education. Longitudinal models of nPEP use include only "on study" nPEP use (i.e., use during follow-up), since nPEP use at baseline reflected prior lifetime use. Logistic regression is used to assess association between nPEP use and sexual risk and substance use, with generalized estimating equation (GEE) modeling methods use to account for within subject correlation of the repeated measure of nPEP. All models assessing association of nPEP with risk behaviors or drug and alcohol risk were adjusted for site, study visit, education and race/ethnicity.

Survival analysis with discrete, twice yearly visits was used to assess the association between HIV seroconversion and time dependent use of nPEP, stratified by site and adjusted for visit. A paired $t$-test was used to assess change in disinhibition perception score before and after nPEP, where a numeric score was constructed from the 6 point Likert agreement scale (score $1=$ strongly disagree to 6 = strongly agree).

Association between nPEP use at the previous visit, willingness and the sexual risk behavior outcome SDUA used logistic regression with GEE methods, adjusting for site, study visit, education and race/ethnicity. These analyses include only risk behaviors following the 6 month visit, as on-study nPEP use is first reported at 6 months.

\section{Results}

\section{Extent of nPEP Use in EXPLORE Participants}

Amongst the 4,295 participants in Explore, 315 people reported using nPEP on at least one occasion, 81 (1.9\%) people reported nPEP use prior to enrollment, 256 people $(6.3 \%)$ reported nPEP use during study participation, 44 of whom reported nPEP use at more than one visit. No association between nPEP use and race/ethnicity, age and education was found at baseline or during follow-up (Table 1). The two nPEP sites, Boston and San Francisco, had higher levels of nPEP use compared to the other 4 sites at baseline $(3.6 \%$ vs. $\left.1.0 \%, \chi^{2}=31, P<0.001\right)$ and during follow-up $(2.1 \%$ vs. $1.0 \%$ of visits, $\left.\chi^{2}=30, P<0.001\right)$.

\section{nPEP Knowledge, Willingness and Access}

At baseline, 2,037 participants (47.5\%) had heard of nPEP, with higher awareness reported at nPEP sites (62\%) relative to non-nPEP sites (40\%). NPEP sites had more recognition through advertising (23\% vs. $8 \%$ at non nPEP sites) and newspaper (62\% vs. $48 \%$ ); whereas non-nPEP sites had information came directly from health care providers (18\% in nPEP sites vs. $29 \%$ at non nPEP sites). However there was no difference in willingness to use nPEP: at nPEP sites $69 \%$ of MSM reported at baseline they were "very likely" to use nPEP after a high risk exposure, compared to $66 \%$ at non-nPEP sites $\left(\chi^{2}=5.6, P=0.06\right)$. Perceived access to nPEP was only modestly better in the nPEP sites of Boston and San Francisco, where $58 \%$ of participants thought they would have ready access to nPEP compared to $53 \%$ at the sites with no nPEP program $\left(\chi^{2}=8.7, P=0.003\right)$. 
Table 1 Baseline and followup nPEP use and participant demographics

\begin{tabular}{|c|c|c|c|c|}
\hline & \multicolumn{2}{|c|}{ Ever used nPEP prior to study } & \multicolumn{2}{|c|}{ Ever used nPEP during study } \\
\hline & $81 / 4289$ & $1.9 \%$ & $256 / 3819$ & $6.7 \%$ \\
\hline \multicolumn{5}{|l|}{ Overall } \\
\hline \multicolumn{5}{|l|}{ Site } \\
\hline Boston & $15 / 728$ & $2.1 \%$ & $49 / 650$ & $7.5 \%$ \\
\hline Chicago & $4 / 624$ & $0.6 \%$ & $30 / 527$ & $5.7 \%$ \\
\hline Denver & $3 / 726$ & $0.4 \%$ & $20 / 683$ & $2.9 \%$ \\
\hline NYBC & $11 / 734$ & $1.5 \%$ & $41 / 657$ & $6.2 \%$ \\
\hline San Francisco & $37 / 734$ & $5.0 \%$ & $76 / 607$ & $12.5 \%$ \\
\hline Seattle & $11 / 743$ & $1.5 \%$ & $40 / 675$ & $5.8 \%$ \\
\hline \multicolumn{5}{|l|}{ Race/Ethnicity } \\
\hline White, not Hispanic & $60 / 3110$ & $1.9 \%$ & $176 / 2792$ & $6.3 \%$ \\
\hline Black, not Hispanic & $5 / 281$ & $1.8 \%$ & $26 / 243$ & $10.7 \%$ \\
\hline Hispanic & $9 / 648$ & $1.4 \%$ & $41 / 566$ & $7.2 \%$ \\
\hline Other & $7 / 249$ & $1.8 \%$ & $13 / 212$ & $6.1 \%$ \\
\hline \multicolumn{5}{|l|}{ Age } \\
\hline $16-19$ & $1 / 93$ & $1.1 \%$ & $5 / 71$ & $7.0 \%$ \\
\hline $20-25$ & $6 / 720$ & $0.8 \%$ & $39 / 617$ & $6.3 \%$ \\
\hline $26-30$ & $12 / 911$ & $1.3 \%$ & $49 / 811$ & $6.0 \%$ \\
\hline $31-35$ & $26 / 909$ & $2.9 \%$ & $58 / 825$ & $7.0 \%$ \\
\hline $36-40$ & $12 / 754$ & $1.6 \%$ & $39 / 675$ & $5.8 \%$ \\
\hline$>40$ & $24 / 902$ & $2.7 \%$ & $66 / 820$ & $8.0 \%$ \\
\hline \multicolumn{5}{|l|}{ Education } \\
\hline High School/GED & $5 / 406$ & $1.2 \%$ & $24 / 330$ & $7.3 \%$ \\
\hline Some College & $19 / 1128$ & $1.7 \%$ & $55 / 986$ & $5.6 \%$ \\
\hline College Degree & $32 / 1855$ & $1.7 \%$ & $109 / 1669$ & $6.5 \%$ \\
\hline Graduate/Professional & $25 / 898$ & $2.8 \%$ & $68 / 833$ & $8.2 \%$ \\
\hline
\end{tabular}

per 100 person years) with no nPEP use (hazard ratio: 0.91, [95\%CI: $0.29,2.86]$ ).

NPEP use occurred more frequently in men with high risk sexual behaviors (Table 3). Those reporting 10 or more partners had almost triple adjusted odds of nPEP use (aOR: 2.9, [95\%CI: 1.9, 4.4]) relative to those reporting zero or one. Significantly increased odds of nPEP use were also seen for men reporting 2-5 and 6-9 partners. Odds of nPEP use increased stepwise with the HIV transmission risk of the reported sexual behaviors: highest odds of nPEP use were found with highest risk behaviors (URA with $\mathrm{HIV}+$, $\mathrm{OR}=6.8$ [95\%CI: 5.0, 9.2], UIA with HIV $+\mathrm{OR}=3.6$ [95\%CI: 2.7, 4.9]); increased odds of nPEP were found with partners of unknown HIV status (URA with HIV-unk, $\mathrm{OR}=3.5$ [95\%CI: 2.7, 4.5]; UIA with HIV-unk, OR $=1.4$ [95\%CI: 1.0, 1.8]). We did not find increased odds of nPEP with unprotected sex with HIV uninfected partners: indeed the trend was for decreased likelihood of nPEP use.

In a multivariable model including all sexual risk behaviors, the highest transmission risk behaviors, URA with HIV+, UIA with HIV+ and URA with HIV-unk had statistically significant higher odds of nPEP use, and those who reported in more than 5 partners were also more likely 
Table 2 Non-occupational Post Exposure Prophylaxis use and illicit drug and alcohol use a All analyses adjusted for site, study visit, race/ethnicity and education

b All analyses include only follow-up data, baseline proportions given for reference

\begin{tabular}{lcccr}
\hline Behavior (last 6 months) & $\begin{array}{l}\text { Proportion } \\
\text { (Baseline) }\end{array}$ & $\mathrm{aOR}^{\mathrm{a}}$ & $(95 \% \mathrm{CI})$ & $P$-value \\
\hline Any non-injection drugs & $64.7 \%$ & 1.50 & $(1.13,1.99)$ & 0.005 \\
Injection drugs & $10.2 \%$ & 2.44 & $(1.69,3.51)$ & $<0.001$ \\
Non injection use of: & & & & \\
Marijuana & $46.3 \%$ & 1.22 & $(0.95,1.58)$ & 0.120 \\
Poppers & $36.6 \%$ & 1.48 & $(1.15,1.91)$ & 0.002 \\
Crack cocaine & $4.2 \%$ & 1.88 & $(1.15,3.09)$ & 0.012 \\
Cocaine(snorted or sniffed) & $19.3 \%$ & 1.27 & $(0.93,1.75)$ & 0.129 \\
Amphetamines & $12.9 \%$ & 2.32 & $(1.74,3.10)$ & $<0.001$ \\
Hallucinogens & $24.0 \%$ & 1.47 & $(1.11,1.94)$ & 0.007 \\
Alcohol & & & & - \\
None & $10.5 \%$ & 1.00 & & 0.51 \\
Light & $46.9 \%$ & 0.89 & $(0.62,1.27)$ & 0.74 \\
Moderate & $32.1 \%$ & 0.93 & $(0.63,1.39)$ & 0.77 \\
Heavy & $10.6 \%$ & 1.09 & $(0.62,1.89)$ & \\
\hline
\end{tabular}

Table 3 Non-occupational Post Exposure Prophylaxis Use during follow-up and HIV sexual risk behaviors

\begin{tabular}{|c|c|c|c|c|c|c|c|}
\hline \multirow[t]{2}{*}{ Behavior in last 6 months } & \multirow{2}{*}{$\begin{array}{l}\text { Proportion in } \\
\text { category (Baseline) }^{\mathrm{b}}\end{array}$} & \multicolumn{3}{|c|}{ Univariate Models $^{\mathrm{a}}$} & \multicolumn{3}{|c|}{ Multivariable model ${ }^{\mathrm{a}}$} \\
\hline & & OR & $95 \% \mathrm{CI}$ & $P$-value & $\mathrm{aOR}$ & $95 \% \mathrm{CI}$ & $P$-value \\
\hline URA with HIV- & $32.1 \%$ & 1.28 & $(0.99,1.66)$ & 0.062 & 1.27 & $(0.91,1.77)$ & 0.156 \\
\hline UIA with HIV- & $32.9 \%$ & 0.77 & $(0.58,1.01)$ & 0.058 & 0.59 & $(0.42,0.83)$ & 0.002 \\
\hline URA with HIV-unk & $25.1 \%$ & 3.47 & $(2.68,4.48)$ & $<0.001$ & 2.67 & $(1.90,3.75)$ & $<0.001$ \\
\hline UIA with HIV-unk & $31.7 \%$ & 1.36 & $(1.04,1.77)$ & 0.023 & 0.69 & $(0.49,0.98)$ & 0.037 \\
\hline URA with HIV+ & $5.9 \%$ & 6.79 & $(5.01,9.19)$ & $<0.001$ & 3.37 & $(2.13,5.31)$ & $<0.001$ \\
\hline UIA with HIV+ & $10.5 \%$ & 3.63 & $(2.67,4.94)$ & $<0.001$ & 1.87 & $(1.19,2.85)$ & 0.007 \\
\hline \multicolumn{8}{|c|}{ Number of male sex partners } \\
\hline $0-1$ & $7.2 \%$ & 1.00 & & - & 1.00 & & - \\
\hline $2-5$ & $32.5 \%$ & 1.66 & $(1.07,2.57)$ & 0.02 & 1.44 & $(0.93,2.27)$ & 0.10 \\
\hline $6-9$ & $23.0 \%$ & 2.29 & $(1.45,3.60)$ & $<0.001$ & 1.75 & $(1.11,2.76)$ & 0.016 \\
\hline $10+$ & $37.3 \%$ & 2.91 & $(1.91,4.44)$ & $<0.001$ & 1.77 & $(1.27,2.78)$ & 0.013 \\
\hline
\end{tabular}

$\mathrm{URA}=$ any unprotected receptive anal sex, UIA $=$ any unprotected insertive anal sex

HIV $-=$ HIV uninfected partner, HIV-unk = partner of unknown HIV status, HIV $+=$ HIV infected partner

${ }^{a}$ All analyses adjusted for site, race/ethnicity, education and study visit

b All analyses include only follow-up data, baseline proportions given for reference

to use nPEP. Those reporting putatively lower risk behaviors: UIA with HIV- and UIA with HIV-unk were markedly less likely to report nPEP use, after adjustment for all other risk behaviors, with adjusted odds ratios of 0.58 [95\%CI: $0.42,0.81]$ and 0.69 [95\%CI: $0.54,0.98$ ], respectively.

Perception of Disinhibition and nPEP Use

The perception that nPEP would increase HIV risk behaviors differed between those who had versus hadn't previously used nPEP at baseline: $60 \%$ of prior nPEP users disagreed that availability of nPEP would increase unsafe sex, $58 \%$ of those never using nPEP agreed with this statement $\left(\chi^{2}=10.0, P=0.002\right)$. We examined whether perceptions of nPEP and risk changed as a result of nPEP use: amongst the 231 first time users of nPEP during EXPLORE, we found the mean perception of disinihibition score before nPEP use was 3.32 but after was 2.64 , indicating a significant shift toward disagreeing with the perception that nPEP use increased high risk. behavior (paired $t$-test $=7.7, P<0.001)$. 
Association Between Willingness to Use nPEP, nPEP Use and HIV Risk Behaviors

We examined whether willingness to use nPEP use aligned with actual nPEP use. Those "very likely" to use nPEP following a known high risk exposure at baseline also had higher odds of reporting subsequent nPEP use: aOR $=3.08$ [95\% CI 2.25, 4.20, $P<0.001]$ compared to those who had said "somewhat" or "not very likely". We also found those "very likely" to use nPEP had significantly lower odds of subsequently reporting SDUA (aOR: 0.59 [95\% CI: 0.54, 0.65], Table 4).

High risk sexual behavior was more likely in those who had previously reported nPEP. In men who used nPEP at the prior visit, there were elevated odds of reporting SDUA compared to those who didn't previously use nPEP (aOR: 1.6, [95\%CI: 1.2, 2.1], Table 4). However, when the comparison is restricted to prior participant visits that were potentially eligible for nPEP, i.e. visits where SDUA was reported, the adjusted odds of SDUA was 0.76 [95\%CI: $0.54,1.06]$ in those who had used nPEP compared to those who had not.

\section{Discussion}

Previous studies in San Francisco [17] and amongst vaccine study participants [14] have reported low use of nPEP in the community setting. In the context of pro-active provision of nPEP in 4-day take-home supply kits in a Brazilian study [15], uptake was sharply increased, with $34 \%$ of men reporting beginning a course of PEP following a high risk exposure. Uptake of nPEP at baseline in EXPLORE is consistent with other cross-sectional studies in the US, with only a small fraction of men reporting use of antiretrovirals after a high risk exposure. In the EXPLORE cohort somewhat higher reporting is seen during follow-up, possibly as a result of increased contact with the site during study participation. Increased knowledge and perceived access as well as increased nPEP use was seen in sites with active nPEP programs, suggesting that exposure to nPEP information are factors that could influence increased uptake of this prevention strategy, however the use of nPEP in this high risk cohort is still low. Unlike Liu [15], we found no patterns of association with education.

Knowledge of nPEP was perhaps surprisingly low given the wide spread use of treatment for HIV/AIDS in these cities, with less than half the cohort aware of this use of antiretroviral drugs, and only $40 \%$ in sites without active nPEP programs. Much of the knowledge seems to have been propagated through health care providers and word of mouth; clearly newspapers and advertising were effective in increasing awareness in the nPEP sites. Interestingly, in a subsequent study at the same Boston site, Mayer [13] found even lower knowledge of pre-exposure prophylaxis (PrEP), with $81 \%$ of Boston MSM having not heard about PrEP. In that group, concerns about antiretroviral side effects and behavioral disinhibition were ameliorated with information about side effects and cautions against risk compensation. The increased utilization of nPEP during the EXPLORE study at the San Francisco and Boston sites would suggest that local programs can result in enhanced utilization.

NPEP was not associated with any suggestion of change in seroconversion risk in this cohort, though low rates of nPEP use gave little power to detect an association. NPEP use in this cohort clearly reflected the CDC guidelines in place: nPEP use was most strongly associated with the highest risk HIV behaviors with partners known to be HIVinfected or whose status is unknown. The finding in the multivariable analysis that nPEP is significantly less likely to be used with unprotected insertive sex with HIV uninfected partners or partners of unknown status possibly reflects a subgroup who exclusively practiced risky insertive sex and did not use nPEP because they did not consider themselves at risk. As also reported in other studies, increased uptake of nPEP is found amongst those who use illicit drugs, and those who inject drugs, while alcohol use has no association with nPEP use.

Perception about nPEP use and behavioral disinhibition differed depending on whether the participant had

Table 4 Serodiscordant unprotected sex (SDUA) and association with willingness to use and previous use of non-occupational Post Exposure Prophylaxis

\begin{tabular}{|c|c|c|c|}
\hline nPEP behaviors & $N$ & $\begin{array}{l}\text { Any SDUA } \\
\mathrm{aOR}^{\mathrm{a}}(95 \% \mathrm{CI})\end{array}$ & $P$-value \\
\hline Willingness to use nPEP: Very likely vs. somewhat/not likely to use nPEP after high risk sex & 4091 & $0.59(0.54,0.65)$ & $<0.001$ \\
\hline nPEP use vs. no nPEP use at previous visit & 4091 & $1.62(1.23,2.13)$ & 0.001 \\
\hline nPEP use vs. no nPEP use at previous visit, in men reporting SDUA at previous visit. & 2898 & $0.76(0.54,1.06)$ & 0.11 \\
\hline
\end{tabular}

SDUA - unprotected anal sex with an HIV infected or unknown HIV status partner

${ }^{\text {a }}$ All analyses adjusted for site, race/ethnicity, education and study visit 
previously used nPEP. For those who had used nPEP prior to the study, the majority did not think the availability of nPEP after high risk exposures would increase the likelihood of unsafe sex. In those who had never used nPEP, the majority view was opposite. Similarly, amongst those using nPEP for the first time during study follow-up the perception that nPEP would increase unsafe sex changes from the "agreement" to "disagreement" range following first nPEP use. The difference in perception seems likely to simply reflect changes in attitudes following actual experience of nPEP - those who have never used nPEP are answering a hypothetical question, whereas those who have used nPEP can answer based on their own experience. Decreased risk was reported after nPEP use in a San Francisco cohort-a report that is consistent with nPEP user's perceptions in our study. Dissonance between hypothetical questions and actual behavior is seen in other situations, for example, in hypothetical vs. actual willingness to be in a vaccine trial [27].

MSM who knew about nPEP and stated they would very likely use nPEP after a known exposure were more likely to use nPEP, demonstrating consistency between knowledge, willingness and actions. However willingness to use nPEP was not an indicator of greater risk, indeed we found willingness was associated with $40 \%$ lower odds of SDUA. Increased knowledge about nPEP and its availability as a prevention strategy did not lead to increased risk in this cohort.

None-the-less, nPEP use does appear to characterize a group with higher HIV risk behaviors. A unique strength of this study is the longitudinal prospective assessment of nPEP where we can assess the effect of nPEP use on subsequent behavior. We found users of nPEP remained a high risk group compared to the cohort as a whole, reporting higher SDUA at subsequent visits. However nPEP use did not appear to lead to increased sexual risk amongst men who reported risk behaviors that would make them eligible for nPEP. Reduction in risk was similarly found after use of nPEP in a prospective study of MSM in San Francisco [18]. This supports other similar reports that nPEP use did not lead to increased risk behavior, but also points to the need for access to repeated nPEP use amongst MSM with the highest exposure risk.

This study only reports attitudes and use of nPEP within a high risk cohort in large urban centers with large MSM populations. The low rate of nPEP use and small number of seroconversions observed gives low power to determine whether nPEP was effective in preventing infection. Risk behaviors and nPEP use were self-reported for the previous 6 month period, and it was not possible to assess whether nPEP use followed or preceded high risk behavior.

This study provides strong evidence that the CDC guidelines for nPEP use were being followed within this cohort of high risk men who have sex with men, although uptake was not high. The perception in the non-nPEP users that unsafe sex is made more likely with availability of nPEP points to a negative perception of nPEP as a prevention strategy, potentially a factor associated with its non-use. Our findings suggest that nPEP was not perceived as an alternative to safe sex, nor that the experience of accessing and taking nPEP increased risky sexual behavior. However, within the subset of people who had reported sex behaviors where nPEP use was recommended, previous nPEP use was not associated with higher odds of high risk sex, thus allaying fears that availability of nPEP would lead to an increase in high risk sex.

Acknowledgements We gratefully acknowledge the contributions of the EXPLORE study participants and the EXPLORE Study Team.

This work was supported by the HIV Network for Prevention Trials and sponsored by the US National Institute of Allergy and Infectious Diseases and the National Institute on Alcohol Abuse and Alcoholism, the National Institute of Child Health and Human Development, the National Institute on Drug Abuse, the National Institute of Mental Health, and the Office of AIDS Research of the National Institutes of Health, Department of Health and Human Services, through contract N01 AI35176 with ABT Associates Inc; contract N01 AI45200 and U01 AI068617 with the Fred Hutchinson Cancer Research Center; cooperative agreement U01 AI46749 and N01 AI35173 with Family Health International and subcontracts with the Denver Public Health, the Fenway Community Health Center, the Howard Brown Health Center, the New York Blood Center, the Public Health Foundation Inc., and the University of Washington, in addition to cooperative agreement U01 AI48040 to the Fenway Community Health Center; cooperative agreement U01 AI48016 to Columbia University (including a subagreement with the New York Blood Center); cooperative agreement U01 AI47981 to the University of Washington; cooperative agreement U01 AI47995 to the University of California, San Francisco.

Open Access This article is distributed under the terms of the Creative Commons Attribution Noncommercial License which permits any noncommercial use, distribution, and reproduction in any medium, provided the original author(s) and source are credited.

\section{References}

1. Cardo DM, Culver DH, Ciesielski CA, et al. A case-control study of HIV seroconversion in health care workers after percutaneous exposure. Centers for Disease Control and Prevention Needlestick Surveillance Group. N Engl J Med. 1997;337(21):1485-90.

2. Cohen MS, Gay C, Kashuba AD, Blower S, Paxton L. Narrative review: antiretroviral therapy to prevent the sexual transmission of HIV-1. Ann Intern Med. 2007;146(8):591-601.

3. Smith DK, Grohskopf LA, Black RJ, et al. Antiretroviral postexposure prophylaxis after sexual, injection-drug use, or other nonoccupational exposure to HIV in the United States: recommendations from the U.S. Department of Health and Human Services. MMWR Recomm Rep. 2005;54(RR-2):1-20.

4. Otten RA, Smith DK, Adams DR, et al. Efficacy of postexposure prophylaxis after intravaginal exposure of pig-tailed macaques to a human-derived retrovirus (human immunodeficiency virus type 2). J Virol. 2000;74(20):9771-5. 
5. Schechter M, do Lago RF, Mendelsohn AB, Moreira RI, Moulton LH, Harrison LH. Behavioral impact, acceptability, and HIV incidence among homosexual men with access to postexposure chemoprophylaxis for HIV. J Acquir Immune Defic Syndr. 2004;35(5):519-25.

6. Tsai CC, Emau P, Follis KE, et al. Effectiveness of postinoculation (R)-9-(2-phosphonylmethoxypropyl) adenine treatment for prevention of persistent simian immunodeficiency virus SIVmne infection depends critically on timing of initiation and duration of treatment. J Virol. 1998;72(5):4265-73.

7. Tsai CC, Follis KE, Sabo A, et al. Prevention of SIV infection in macaques by (R)-9-(2-phosphonylmethoxypropyl)adenine. Science. 1995;270(5239):1197-9.

8. Van Rompay KK, McChesney MB, Aguirre NL, Schmidt KA, Bischofberger N, Marthas ML. Two low doses of tenofovir protect newborn macaques against oral simian immunodeficiency virus infection. J Infect Dis. 2001;184(4):429-38.

9. Van Rompay KK, Miller MD, Marthas ML, et al. Prophylactic and therapeutic benefits of short-term 9-[2-(R)-(phosphonomethoxy)propyl]adenine (PMPA) administration to newborn macaques following oral inoculation with simian immunodeficiency virus with reduced susceptibility to PMPA. J Virol. 2000;74(4):1767-74.

10. Rep MMMW. Case-control study of HIV seroconversion in health-care workers after percutaneous exposure to HIV-infected blood-France, United Kingdom, and United States, January 1988-August 199444. 1995/12/22 ed. 1995:929-33.

11. Limb S, Kawsar M, Forster GE. HIV post-exposure prophylaxis after sexual assault: the experience of a sexual assault service in London. Int J STD AIDS. 2002;13(9):602-5.

12. Linden JA, Oldeg P, Mehta SD, McCabe KK, LaBelle C. HIV postexposure prophylaxis in sexual assault: current practice and patient adherence to treatment recommendations in a large urban teaching hospital. Acad Emerg Med. 2005;12(7):640-6.

13. Mayer KH, Mimiaga MJ, Cohen D, et al. Tenofovir DF plus lamivudine or emtricitabine for nonoccupational postexposure prophylaxis (NPEP) in a Boston Community Health Center. J Acquir Immune Defic Syndr. 2008;47(4):494-9.

14. Ackers ML, Buchbinder S, McKirnan D, et al. Post-exposure prophylaxis among HIV-uninfected participants in a phase III HIV vaccine efficacy trial. Paper presented at: XIV International Conference on AIDS; July, 2002; Barcelona, Spain.

15. Liu AY, Kittredge PV, Vittinghoff E, et al. Limited knowledge and use of HIV post- and pre-exposure prophylaxis among gay and bisexual men. J Acquir Immune Defic Syndr. 2008;47(2):241-7.

16. Management of possible sexual, injecting-drug-use, or other nonoccupational exposure to HIV, including considerations related to antiretroviral therapy. Public Health Service statement. Centers for Disease Control and Prevention Sep 25 1998. 10575987 (Print).

17. Martin JN, Roland ME, Bamberger JD, et al. Post-exposure prophylaxis (PEP) for sexual exposure to HIV does not lead to increases in high risk behavior: the San Francisco PEP Project Paper presented at: 8th Conference on Retroviruses and Opportunistic Infections; February 4-8, 2001; Chicago, Illinois.

18. Martin JN, Roland ME, Neilands TB, et al. Use of postexposure prophylaxis against HIV infection following sexual exposure does not lead to increases in high-risk behavior. AIDS. 2004;18(5):787-92.

19. Golub SA, Rosenthal L, Cohen DE, Mayer KH. Determinants of high-risk sexual behavior during post-exposure prophylaxis to prevent HIV infection. AIDS Behav. 2008;12(6):852-9.

20. Schechter M, Lago R, Moreira RI, Mendelsohn AB, Harrison L. Behavioral impact of the availability of post-sexual-exposure chemoprophylaxis (PEP) for HIV: a prospective cohort study. 1st IAS Conference on Pathogenesis and Treatment. Vol Buenos Aires, Argentina; 2001.

21. Van der Straten A, Gomez C, Saul J, Padian N. Awareness of PEP and viral suppressive therapy have little effect on sexual risk behavior in heterosexual HIV-discordant couples. XII International Conference on AIDS. Vol Geneva, Switzerland; 1998.

22. Waldo CR, Stall RD, Coates TJ. Is offering post-exposure prevention for sexual exposures to HIV related to sexual risk behavior in gay men? AIDS. May 26 2000;14(8):1035-9.

23. Koblin B, Chesney M, Coates T. Effects of a behavioural intervention to reduce acquisition of HIV infection among men who have sex with men: the EXPLORE randomised controlled study. Lancet. 2004;364(9428):41-50.

24. Koblin BA, Chesney MA, Husnik MJ, et al. High-risk behaviors among men who have sex with men in 6 US cities: baseline data from the EXPLORE Study. Am J Public Health. 2003;93(6):92632.

25. Chesney MA, Koblin BA, Barresi PJ, et al. An individually tailored intervention for HIV prevention: baseline data from the EXPLORE Study. Am J Public Health. 2003;93(6):933-8.

26. Kamb ML, Fishbein M, Douglas JM Jr, et al. Efficacy of risk-reduction counseling to prevent human immunodeficiency virus and sexually transmitted diseases: a randomized controlled trial. Project RESPECT Study Group. JAMA. 1998;280(13): 1161-7.

27. Buchbinder SP, Metch B, Holte SE, Scheer S, Coletti A, Vittinghoff E. Determinants of enrollment in a preventive HIV vaccine trial: hypothetical versus actual willingness and barriers to participation. J Acquir Immune Defic Syndr. 2004;36(1):604-12. 